\title{
Formação de Médicos Especialistas no SUS: Descrição e Análise da Implementação do Programa Nacional de Apoio à Formação de Médicos Especialistas em Áreas Estratégicas (Pró-Residência)
}

\author{
Training of Medical Specialists in SUS: \\ Description and Analysis of the \\ Implementation of the National Programme \\ to Support the Training of Specialists in \\ Strategic Areas (Pro-Residencia)
}

Helena Lemos Petta

\begin{abstract}
PALAVRAS-CHAVE:
- Educação Médica;

- Sistema Único de Saúde;

- Formulação de Políticas;

- Políticas Públicas;

- Gestão em Saúde;

- Estratégias.
\end{abstract}

KEYWORDS:

- Medical Education;

- Unified Health System;

- Policy Formulation;

- Public Policy;

- Management in Health;

- Strategies

Recebido em: 01/09/2012

Aprovado em: 26/02/2013

REVISTA BRASILEIRA DE EDUCAÇÃo MÉDICA

Este artigo descreve e analisa os objetivos, estratégias, problemas e dificuldades na implementação do Programa Nacional de Apoio à Formação de Médicos Especialistas em Áreas Estratégicas (Pró-Residência). Com base na descrição dos resultados dos dois Editais lançados em outubro de 2009 e nos avanços relacionados aos objetivos da política proposta, foi realizada uma pesquisa, sendo utilizadas abordagens qualitativas e quantitativas. A abertura de vagas pelo programa indicou melhor distribuição geográfica e priorização de especialidades estratégicas para o SUS, e os projetos de apoio matricial revelaram capacidade de gerar mudança no modelo de formação, iniciando uma nova relação entre as necessidades do sistema de saúde e a formação médica especializada. Conclui-se que a estratégia do apoio matricial constitui um modelo de política pública mais potente para gerar as mudanças necessárias na formação médica especializada. Porém, outras ações estruturantes para a residência médica, como um novo marco regulatório e Diretrizes Curriculares Nacionais, combinadas à implementação de políticas para gestão do trabalho médico especializado no SUS, são fundamentais para contribuir com a efetivação da política proposta.

This paper describes and analyzes the objectives, strategies, problems and difficulties surrounding the implementation of the National Support Program for the Training of Specialists in Strategic Areas known as "PRO-RESIDENCIA". From the description of the results of two notices issued in October 2009 and the progress toward the objectives of the proposed policy, we conducted research employing both qualitative and quantitative approaches. Results showed that the vacancies generated by the program reflected a better geographical distribution and the prioritization of strategic expertise for the SUS. Matrix support projects revealed the training model's capacity to generate change, redefining the relationship between the healthcare system's needs and specialized medical training. We conclude that the strategy of using the support matrix serves as a more powerful model for public policy to generate the necessary changes in specialist medical training. Other structural initiatives for residency are, however, fundamental to the effectiveness of the proposed policy, such as a new regulation and national curriculum guidelines combined with the implementation of policies for the management of medical specialists in the public health system. 


\section{INTRODUÇÃO}

Este artigo descreve e analisa dados preliminares da implementação de uma política pública voltada para induzir a abertura de vagas e programas de residência médica em áreas prioritárias para o Sistema Único de Saúde. Combinado à ampliação de vagas, buscou-se induzir a mudança de modelo dos programas de residência médica segundo uma concepção que prioriza as necessidades sociais, a integração dos processos formativos aos serviços assistenciais organizados em redes de atenção em saúde e o desenvolvimento de habilidades e competências para a formação do especialista.

A formação médica especializada, por meio da residência médica, embora institucionalizada no País há mais de 30 anos e financiada pelo poder público, nunca havia sido objeto de formulação de políticas públicas específicas, carecendo, portanto, de mecanismos de articulação entre necessidades de profissionais e oferta de formação. Durante esse período, prevaleceram interesses coorporativos e de mercado pela ausência de ações regulatórias para a residência médica entendidas como "regras, orientações, medidas de controle e valoração que possibilitam o exercício do controle social em atividades de serviço público [...] permitindo a adequação necessária às diferentes circunstâncias que se configuram “1 (p. 27-40) .

Em outubro de 2009, foi lançado pela Secretaria de Gestão do Trabalho e da Educação na Saúde, do Ministério da Saúde (MS), e pela Secretaria de Educação Superior, do Ministério da Educação (MEC), o Programa Nacional de Apoio à Formação de Médicos Especialistas em Áreas Estratégicas (Pró-Residência) $^{2}$ com o objetivo de favorecer a formação de médicos especialistas, na modalidade residência médica, em especialidades e regiões prioritárias do País, definidas em comum acordo com gestores do Sistema Único de Saúde. Esse programa busca articular e promover a interlocução entre as demandas do sistema de saúde, focadas na organização de Redes de Atenção à saúde, e as potencialidades das instituições formadoras. O Pró-Residência vem produzindo um conjunto de transformações que merecem ser investigadas, conforme o objetivo deste estudo.

O objetivo geral deste estudo é descrever e analisar os objetivos e estratégias, problemas e dificuldades na implementação do Programa Nacional de Apoio à Formação de Médicos Especialistas em Áreas Estratégicas (Pró-Residência).

Como objetivos específicos, o estudo pretende descrever e analisar os resultados dos dois Editais lançados em outubro de 2009 e os avanços relacionados aos objetivos da política proposta.

\section{MÉTODOS}

$\mathrm{Na}$ coleta de material para a pesquisa foram analisados dados secundários de revisão bibliográfica sobre o tema e foi realizada análise documental.

Na revisão bibliográfica, consultou-se literatura nacional e internacional sobre os temas relativos a Políticas Públicas, Políticas de Saúde, Políticas para Formação de Recursos Humanos em Saúde, Trabalho Médico, Educação Médica e Residência Médica.

Foram também estudados, para análise documental, os documentos produzidos pela Comissão Interministerial da Gestão da Educação na Saúde ${ }^{3}$, por meio da Subcomissão de Estudos e Avaliação das Necessidades de Médicos Especialistas no Brasil ${ }^{4}$, bem como as Portarias e Editais ${ }^{5,6}$ lançados. Também foram analisados os projetos enviados ao Departamento da Gestão da Educação na Saúde (Deges), bem como um banco de dados disponibilizado por esse Departamento.

$\mathrm{Na}$ análise do material foram utilizadas abordagens qualitativas e quantitativas, produzindo triangulações ${ }^{7,8}$

$\mathrm{Na}$ análise quantitativa foi realizada estatística descritiva dos dados encontrados a partir dos resultados de dois Editais lançados pelo programa. $\mathrm{Na}$ análise qualitativa, buscou-se descrever e analisar a construção e a implementação do programa com base em dois componentes principais: capacidade de indução de abertura de vagas e capacidade de mudança de modelo de formação.

Para tanto, foram elaboradas duas questões principais:

- Os resultados dos Editais 07 e 08/2009 indicam melhor distribuição geográfica e priorização de especialidades estratégicas para o SUS, conforme os objetivos do programa?

- Os projetos aprovados pelo Edital 08/2009 contêm elementos que configuram uma nova relação entre as necessidades do sistema de saúde e a formação médica especializada?

Para a primeira questão foram analisados os resultados dos Editais verificando-se a distribuição dos projetos aprovados por área geográfica (regiões do Brasil) e por especialidades médicas. Em seguida, após o agrupamento das especialidades em linhas de cuidado, também se analisou a distribuição de projetos aprovados por região geográfica dentro de cada linha de cuidado.

Para responder à segunda questão, a pesquisadora optou por analisar os projetos aprovados do Edital 08/2009 pela possibilidade de melhor categorização de seus conteúdos. Para isto, buscou-se analisar os projetos segundo três eixos principais: 
- Referência à necessidade de formação na especialidade segundo as necessidades de saúde;

- Grau de envolvimento da gestão da saúde do município ou estado na proposição;

- Referência a outros cenários de formação, contidos na linha de cuidados em que se insere a especialidade.

Esta pesquisa foi realizada apenas com documentos e dados de livre acesso, públicos e divulgados, não necessitando, portanto, de aprovação pelo Comitê de Ética.

\section{RESULTADOS E DISCUSSÃO}

Para o Edital 07/2009 foram enviados projetos de 72 instituições, com cerca de 960 bolsas solicitadas, em todas as áreas de intervenção. Foram concedidas 785 bolsas, conforme publicação no Diário Oficial da União de 06/01/2010.

O Edital estabelecia que as instituições poderiam pleitear bolsas para vagas já credenciadas pela CNRM ou para vagas ainda não existentes (novos programas ou expansão). Os projetos foram selecionados segundo os critérios estabelecidos no Edital. A Comissão de Seleção foi composta por membros do Ministério da Saúde e da Educação, áreas técnicas de ambos os ministérios e médicos especialistas das áreas prioritárias convidados.

Para melhor visualização dos resultados, as especialidades foram agrupadas segundo áreas de intervenção, conforme previsto no Edital, com e a respectiva correspondência (Quadro 1).

\section{QUADRO 1}

Área de intervenção e especialidades correspondentes

\begin{tabular}{|c|c|}
\hline Área de intervenção & Especialidade/área de atuação \\
\hline Atenção básica & Medicina de Família e Comunidade \\
\hline Urgência & $\begin{array}{l}\text { Medicina Intensiva, Anestesiologia, } \\
\text { Neurologia, Neurocirurgia, Ortopedia e } \\
\text { Traumatologia/ } \\
\text { Medicina de Urgência e Cirurgia do Trauma }\end{array}$ \\
\hline Área Básica & Clínica Médica e Cirurgia Geral \\
\hline Criança & Pediatria/Neonatologia \\
\hline Outras & $\begin{array}{l}\text { Outras especialidades não prioritárias, } \\
\text { porém com escassez na região proponente }\end{array}$ \\
\hline Mulher & Ginecologia e Obstetrícia \\
\hline Mental & $\begin{array}{l}\text { Psiquiatria/Psiquiatria da Infância e } \\
\text { Adolescência }\end{array}$ \\
\hline Câncer & $\begin{array}{l}\text { Cancerologia Clínica, Cancerologia } \\
\text { Cirúrgica, } \\
\text { Radioterapia e Patologia }\end{array}$ \\
\hline Idoso & Geriatria \\
\hline
\end{tabular}

A análise dos dados referentes às bolsas para vagas selecionadas no Edital 07/2009 mostra que, de maneira geral, a iniciativa teve caráter redistributivo em relação à tendência histórica de concentração de vagas na Região Sudeste. Somando-se os percentuais de bolsas distribuídas nas regiões prioritárias (Norte, 7\%; Nordeste, 40\%; Centro-Oeste, 13\%), temos que $60 \%$ das vagas foram de regiões consideradas prioritárias. O restante foi dividido entre as regiões Sudeste (33\%) e Sul (7\%) (Figura 1).

\section{Figura 1}

Distribuição de vagas/bolsas por região $(\mathrm{N}=785)$ Pró-Residência - Edital 07/2009

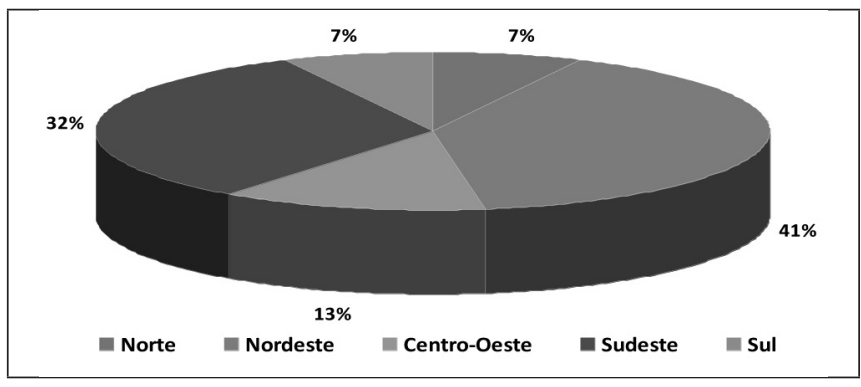

Fonte: Deges/SGTES/MS.

A distribuição das bolsas segundo as áreas prioritárias (Figura 2) destaca o volume significativo de vagas para programas de Medicina de Família e Comunidade (272 vagas). A figura mostra, ainda, o expressivo número de vagas selecionadas para a área de urgência, reconhecidamente crítica em muitos Estados das regiões prioritárias. Esta divisão mostra também que as especialidades básicas (Clínica Médica e Cirurgia Geral) ocupam o terceiro lugar na distribuição de vagas.

\section{Figura 2}

Distribuição de vagas/bolsas por áreas de intervenção ( $\mathrm{N}=785)$ - Pró-Residência Edital 07/2009

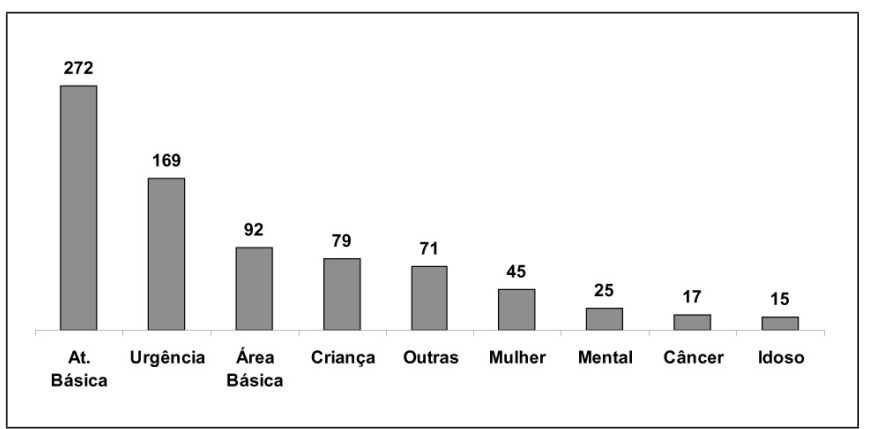


A seleção dos projetos do Edital 08/2009 foi realizada em duas etapas, conforme descrito no Edital. A primeira consistiu na seleção de pré-projetos enviados por instituições que requeriam apoio matricial, com base na descrição das necessidades regionais de formação na especialidade. A segunda etapa constou da seleção de projetos enviados pelas instituições pré-aprovadas na primeira etapa em conjunto com instituições matriciadoras, convidadas pelo Ministério da Saúde.

Para viabilizar as duas etapas do Edital 08/2009, foram realizadas diversas reuniões com especialistas convidados em todo o País, indicados pelas áreas técnicas do Ministério da Saúde, para apoiar a estratégia de apoio matricial. Esses especialistas foram chamados a contribuir na definição de parâmetros para a participação de instituições com perfil para oferecer o necessário matriciamento. Foi ainda solicitado o apoio técnico da Organização Panamericana de Saúde - Representação Brasil (Opas-Brasil) e da Fundação Cearense de Pesquisa e Cultura (FCPC), que firmaram instrumentos de financiamento e prestaram cooperação técnica, na modalidade carta-acordo, para que os pré-projetos fossem analisados em sua viabilidade e pudessem ser iniciados ainda em 2011.

Os especialistas convidados participaram, inicialmente, de reuniões técnicas na SGTES/MS, em Brasília, com vistas à elaboração de parâmetros e critérios quantitativos e qualitativos para identificar instituições matriciadoras nas áreas prioritárias para o SUS. Em um primeiro momento, juntamente com a equipe técnica do Pró-Residência, foram identificados critérios orientadores gerais para identificação/seleção das instituições matriciadoras, entre os quais se destacam:

- Ter programa de residência médica na especialidade reconhecida pelo MEC;

- Possuir credenciamento definitivo do programa de residência da especialidade perante o MEC;

- Apresentar histórico de comprometimento com a implantação da política pública na linha de cuidados em que se insere a especialidade;

- Possuir um corpo de preceptores com residência ou título de especialista;

- Utilizar ferramentas de comunicação a distância para interlocução e segunda opinião formativa em sua prática de ensino.

Estabelecidos esses critérios, foi acordado que os especialistas de cada área, em conjunto com a área técnica do MS, deveriam discutir e elaborar os critérios específicos que orien- tariam a escolha das instituições matriciadoras nos novos programas de residência médica a serem implantados nas diferentes regiões do País.

Foram convidadas 16 instituições matriciadoras, que, prontamente, aceitaram participar do projeto, denominado Termo de Adesão. Duas delas tiveram dificuldades no cumprimento do cronograma. Ao final, participaram 14 instituições: Universidade de São Paulo (USP); Universidade Estadual de Campinas (Unicamp); Universidade Federal de São Paulo (Unifesp); Universidade Federal da Bahia (UFBA); Universidade Federal do Ceará (UFC); Universidade Federal do Rio de Janeiro (UFRJ); Universidade Federal de Minas Gerais (UFMG); Instituto Materno Infantil de Pernambuco (Imip); Fundação Antonio Prudente - Hospital do Câncer - AC Camargo (SP); Escola de Saúde Mental do Rio de Janeiro (Esam); Pontifícia Universidade Católica do Paraná (PUC-PR); Hospital Odilon Behrens (MG); Grupo Hospitalar Conceição (GHC-RS); Secretaria Municipal de Saúde de Fortaleza (SMS-Fortaleza-CE).

A Comissão de Seleção inicialmente selecionou 71 pré-projetos enviados por instituições que requeriam apoio matricial, com vistas à elaboração de projetos em conjunto com instituições matriciadoras.. Após visitas das instituições matriciadoras às instituições pré-selecionadas, realizadas in loco para análise da viabilidade dos pré-projetos, foram elaborados os projetos finais em estreita cooperação matriciada-matriciadora. Conforme a Portaria 08/2010, publicada em 26/11/2010 no Diário Oficial da União, foram aprovados 60 projetos.

\section{Figura 3}

Distribuição dos projetos aprovados por região Edital 08/2009

№ Total - Região (60)

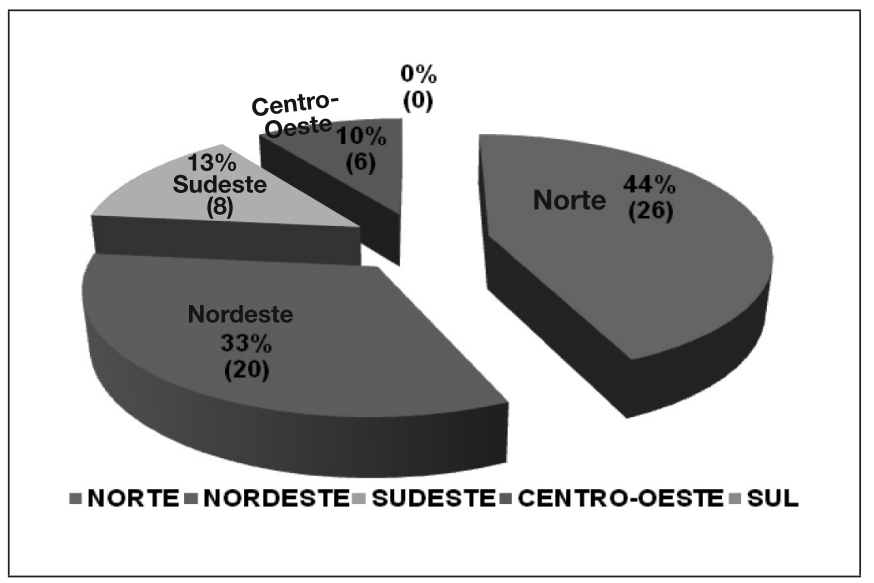

Fonte: Deges/SGTES/MS. 
Entre os projetos aprovados, predominam as regiões Norte $(44 \%)$ e Nordeste (33\%), como indicado na Figura 3. O Sudeste aparece com $13 \%$, com três projetos referentes a universidades federais em processo de implantação da residência médica, e o Centro-Oeste com 10\%. Nenhum projeto foi aprovado na Região Sul.

Entre as especialidades, a que teve maior número de projetos aprovados foi a de Medicina de Família e Comunidade. Em segundo lugar, a Psiquiatria aparece com oito projetos e na sequência vêm os projetos da área básica Clínica Médica.

A Figura 4 ilustra os Estados de origem de matriciadores e matriciadas aprovadas pelo Edital 08/2009.

É importante destacar que o apoio matricial permitirá a abertura de PRM em Estados e especialidades que não exis- tiam até então. O Quadro 2 apresenta as especialidades e Estados em que não havia programas de formação na modalidade residência médica e que foram selecionados para a implantação de programas, via apoio matricial.

Em resposta às demandas apresentadas para o apoio matricial, foram descritas diversas iniciativas, entre as quais se destacam:

- Participação ativa na organização das equipes responsáveis pela preceptoria/capacitação e treinamento dos preceptores;

- Segunda opinião formativa/discussão de casos clínicos com recursos de tecnologias a distância, com apoio do telessaúde;

- Interlocução com o gestor municipal para garantia de cenário em rede ambulatorial/Samu/CAPS/AB;

Figura 4

Instituições Matriciadoras e Estados Matriciados - Edital 08/2009

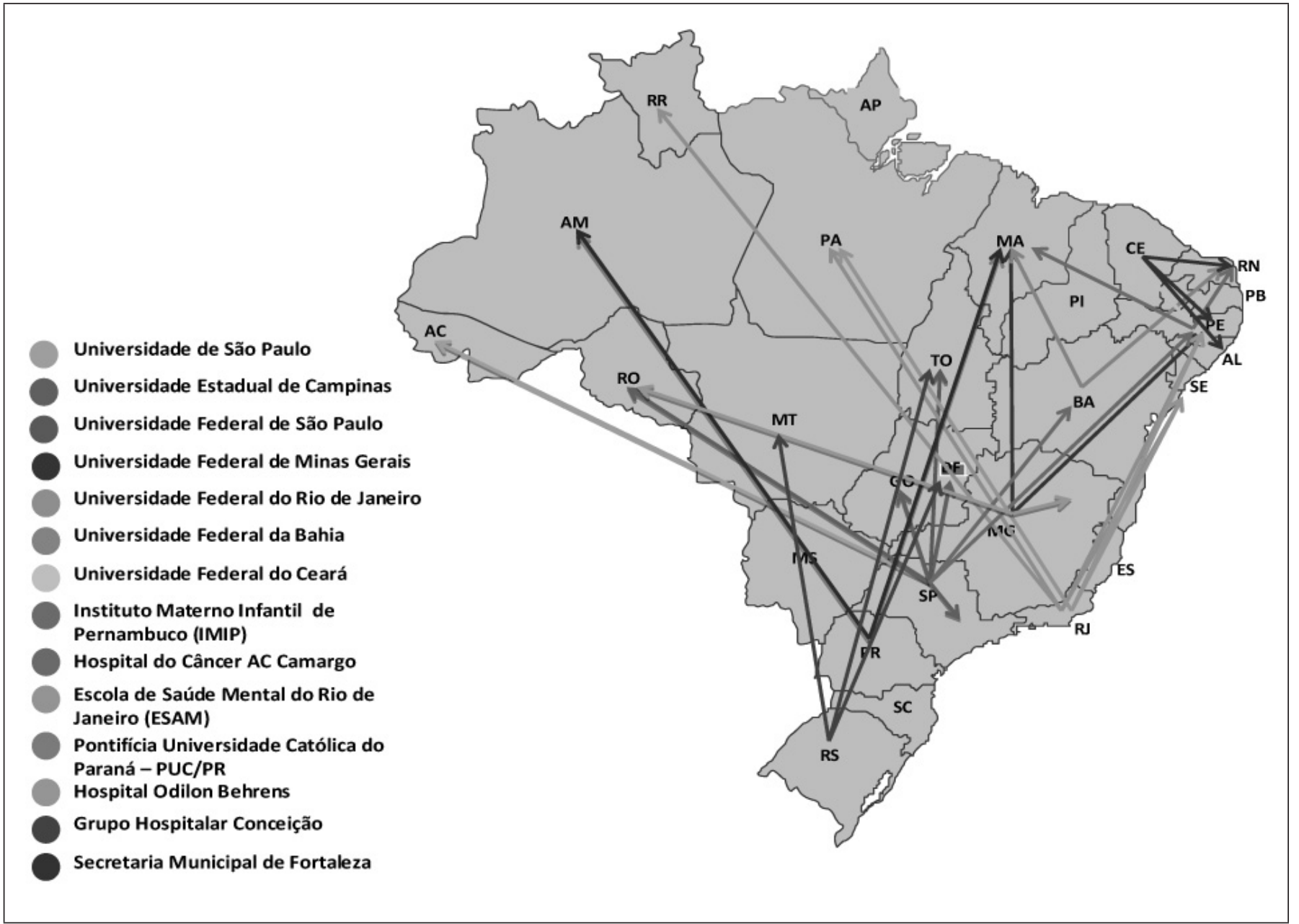

Fonte: DEGES/SGTES/MS. 


\section{QUADRO 2}

Especialidades e Estados apoiados pelo Edital 08/2009 em que não havia oferta de formação via residência médica

\begin{tabular}{|ll|}
\hline $\begin{array}{l}\text { Especialidade/ } \\
\text { área de atuação apoiada }\end{array}$ & Estado \\
\hline Clínica Médica & Tocantins \\
Cirurgia Geral & Tocantins \\
Pediatria & Tocantins \\
Anecologia e Obstetrícia & Tocantins \\
Cancerologia Cirúrgica & Acre, Roraima \\
Cancerologia Clínica & Bahia \\
Geriatria & Acre \\
Medicina de Família e & Maranhão, Pernambuco \\
Comunidade & Amazonas, Tocantins e \\
Medicina Intensiva & Rondônia \\
Neonatologia & Alagoas, Maranhão, Sergipe \\
Padolescência & Acre \\
\hline
\end{tabular}

- Estágios específicos na matriciadora (residentes/preceptores);

- Supervisão presencial na matriciada com reuniões com preceptores e médicos residentes;

- Seminários/simpósios semestrais na matriciada;

- Transmissão por teleconferência de sessões clinicas da matriciadora.

Também se buscou analisar os 60 projetos aprovados pelo Edital 08/2009, segundo três eixos principais:

- Referência à necessidade de formação na especialidade conforme as necessidades de saúde;

- Grau de envolvimento da gestão da saúde do m1unicípio ou Estado na proposição;

- Referência a outros cenários de formação contidos na linha de cuidados em que se insere a especialidade.

Os 60 projetos analisados abordam a necessidade de formação segundo as necessidades de saúde locais, sendo que
24 (40\%) deles mencionam apenas dados epidemiológicos de saúde gerais, como mortalidade geral e cobertura dos serviços de saúde, e 36 (60\%) citam especificamente dados de necessidades relacionadas à especialidade em que se pleiteia apoio.

De maneira geral, os projetos que citam diretamente a busca do apoio para abertura/aprimoramento de PRM em determinada especialidade, relacionada às necessidades de saúde locais, mencionam as iniciativas da gestão para melhoria da assistência na linha de cuidados ou então os elevados índices de mortalidade/letalidade relacionados à área em questão.

Com relação ao grau de envolvimento da gestão da saúde do município ou Estado na proposição, os 60 projetos (100\%) apresentaram adesão da gestão da saúde local. Destes, porém, $38(63,3 \%)$ projetos apenas citam a gestão como parceira, mas não apresentam nenhuma outra iniciativa relacionada à gestão da educação ou do trabalho na linha de cuidados em que se insere a especialidade. Vinte e dois projetos $(36,6 \%)$ apresentam outras iniciativas, como a construção da linha de cuidados relacionada a especialidade, plano de cargos, carreiras e salários, etc.

Por fim, 40 projetos $(66,6 \%)$ fizeram referência a cenários de formação diversos dos habituais, ou seja, cenários não considerados obrigatórios segundo critérios da CNRM (Resolução 02/2006). Apenas 6 projetos (10\%) citaram a diversificação dos cenários como possível de ser construída ao longo do apoio matricial e 14 projetos (23,3\%) não mencionaram essa questão.

Com exceção da área de atuação em Medicina de Urgência, todas as especialidades fazem referência a estágios na Atenção Básica (UBS, ESF). Muitas delas citam o apoio matricial na Atenção Básica como uma estratégia interessante de arranjo possível na residência médica. Destacam-se também os CAPS, Samu, Nasf e Upas como espaços institucionais possíveis para o desenvolvimento da formação médica especializada.

Embora com um curto período de implantação, com base nos eixos analisados, nota-se que os projetos aprovados de apoio matricial revelaram conter elementos que iniciam uma nova relação entre as necessidades do sistema de saúde e a formação médica especializada. A maioria absoluta dos projetos faz referência à necessidade de formação na especialidade segundo as necessidades de saúde, demonstra o envolvimento da gestão da saúde do município ou Estado na proposição e inclui outros cenários de formação, contidos na linha de cuidados em que se insere a especialidade.

\section{CONSIDERAÇÕES FINAIS E CONCLUSÕES}

Primeiramente, é importante destacar o caráter provisório destas conclusões, visto que a construção do projeto é recente, 
e sua implantação ainda está em curso. Novos estudos devem ser realizados sobre o tema, em especial quanto aos futuros desdobramentos desta política, a fim de produzir evidências científicas e adotar novas ações.

No que diz respeito à capacidade de indução de aberturas de vagas pelo programa, os resultados dos Editais indicaram melhor distribuição geográfica e priorização de especialidades estratégicas para o SUS, conforme os objetivos do programa. Porém, um aspecto importante a destacar é que algumas instituições, mesmo após a aprovação pelo Edital e pela CNRM, não conseguiram efetivar suas vagas. Embora essa questão mereça ser mais bem investigada, sabe-se que as especialidades induzidas pelo Pró-Residência não são hoje as mais procuradas pelos recém-formados. É notória a maior procura dos médicos recém-formados por especialidades que agregam procedimentos, elevando, assim, a remuneração, que não necessitam de dedicação e envolvimento contínuo com os pacientes e têm maior prestígio social. Dessa forma, a Medicina de Família e Comunidade e a Pediatria, por exemplo, possuem diversas vagas ociosas no País e foram as especialidades priorizadas pelo programa.

É importante frisar que outras estratégias devem ser desenvolvidas para avançar nesta questão. O Programa Nacional de Reorientação da Formação Profissional em Saúde (Pró-Saúde, 2005) e de incentivo à integração entre ensino e serviços de saúde, como o Programa de Educação pelo Trabalho para Saúde (PET-Saúde, 2010), são iniciativas importantes neste sentido e merecem ser avaliadas quanto a seu impacto. Além disso, ações relacionadas à gestão do trabalho, como melhores salários, plano de carreira e melhores condições de trabalho, também têm relevância nesta questão.

Quanto ao Edital 08/2009, não foi possível analisar sua capacidade de indução de abertura de vagas, visto que ainda está em curso o processo de apoio matricial, que deve ter os primeiros resultados em 2012

Com relação à capacidade do programa de gerar mudança no modelo de formação, os projetos aprovados de apoio matricial revelaram conter elementos que iniciam uma nova relação entre as necessidades do sistema de saúde e a formação médica especializada. A maioria dos projetos, como já apresentado, demonstraram necessidade de formação na especialidade segundo as necessidades de saúde, envolvimento da gestão da saúde do município ou Estado na proposição e referência a outros cenários de formação, contidos na linha de cuidados em que se insere a especialidade. É importante sublinhar que alguns programas de residência sob matriciamento foram identificados, na fase de análise da viabilidade dos pré-projetos, como passíveis de início de funcionamento e recebimento de bolsas em 2011, mas, ainda assim continuarão a receber apoio por no mínimo 12 meses. O número de programas nessas condições é reduzido $(7 / 60)$, estando a maior parte em fase inicial do apoio matricial. Essa continuidade merece melhor investigação em futuros estudos.

É certo que essa indução na mudança de modelo de formação requer outras ações, com especial atenção para a constituição de um novo marco regulatório da residência médica, que contenha elementos importantes desta mudança. A presença de representantes dos gestores estaduais e municipais na composição da CNRM, bem como o atrelamento da abertura de programas a necessidades sociais são itens importantes a considerar, e já existem iniciativas neste sentido. A construção de Diretrizes Curriculares para a Residência Médica que vinculem a formação à construção das Redes de Atenção à Saúde - baseadas em competências a serem adquiridas pelo médico residente, e não mais em conteúdos, ainda que priorizem as necessidades sociais - também constitui um marco na transformação da formação médica especializada. Por fim, a implementação de políticas relacionadas à gestão do trabalho especializado no SUS também é imprescindível à efetivação dos objetivos da política proposta.

\section{REFERÊNCIAS}

1. Conforto G. Descentralização e regulação da gestão de serviços públicos. Rev Admin Pública. 1998;32:27-40.

2. Brasil. Portaria $n^{\circ} 1001$, de 22 outubro de 2009. Instituiu o Programa Nacional de apoio a formação de médicos especialistas em áreas estratégicas. Diário Oficial da União, Brasília, 24 out. 2009.

3. Brasil. Decreto $s / n^{\circ}$, de 20 de junho de 2007. Cria Comissão Interministerial da Gestão da Educação na Saúde e dá outras providências. Diário Oficial da União, Brasília, 21 jun. 2007.

4. Brasil. Portaria Conjunta $n^{\circ} 1$, de 23 de outubro 2007. Institui a Subcomissão de estudos e avaliação das necessidades de médicos especialistas e dá outras providências. Diário Oficial da União, Brasília, 24 out. 2007.

5. Brasil. Edital de convocação nº 7, de 22 de outubro 2009. Programa Nacional de apoio a formação de médicos especialistas em áreas estratégicas. Diário Oficial da União, Brasília, 24 out. 2009.

6. Brasil. Edital de convocação no 8, de 22 de outubro 2009. Programa Nacional de apoio a formação de médicos es- 
pecialistas em áreas estratégicas. Diário Oficial da União, Brasília, 24 out. 2009.

7. Minayo MCS. O desafio do conhecimento. 6 ed. São Paulo: Hucitec; 2000.

8. Minayo MCS, Assis SG, Souza ER, orgs. Avaliação por triangulação de métodos. Rio de Janeiro: Ed Fiocruz; 2005.

\section{ENDEREÇO PARA CORRESPONDÊNCIA}

Helena Lemos Petta

Rua Mauá, 1251, apto 701

Alto da Glória - Curitiba

CEP: 80.030-200 PR

E-mail: helenapetta@hotmail.com

\section{CONFLITO DE INTERESSES}

Declarou não haver. 